\title{
La educación en Europa desde una perspectiva de género*
}

\author{
MARÍA DOLORES BENÍTEZ MÁRQUEZ \\ Departamento Economía Aplicada, Estadística y Econometría, UNIVERSIDAD DE \\ MÁLAGA,ESPAÑA.E-mail: bemarlo@uma.es
}

EUGENIA MARÍA CRUCES PASTOR

Departamento Economía Aplicada, Estadística y Econometría, UNIVERSIDAD DE MÁLAGA,ESPAÑA.E-mail: cruces@uma.es

\section{JULIA DE HARO GARCÍA}

Departamento Economía Aplicada, Estadística y Econometría, UNIVERSIDAD DE MÁLAGA,ESPAÑA. E-mail: haro@uma.es

\section{MARÍA DOLORES SARRIÓN GAVILÁN}

Departamento Economía Aplicada, Estadística y Econometría, UNIVERSIDAD DE MÁLAGA,ESPAÑA. E-mail: dsarrion@uma.es

\section{RESUMEN}

En este trabajo analizamos la educación en países europeos, tanto globalmente como desde una perspectiva de género, y utilizamos la información que se deriva de este análisis para comparar la situación educativa que dichos países presentan con la que observamos en diversos indicadores que reflejan otros aspectos socioeconómicos de los mismos. Para el análisis multidimensional del estado de la educación teniendo en cuenta el género utilizamos la técnica exploratoria de Análisis Factorial Múltiple con el objetivo de analizar globalmente las posiciones relativas de los países considerados en lo que a educación se refiere. La situación educativa de estos países, resumida mediante los primeros factores del análisis factorial, se combina con la que proporcionan diversos indicadores socioeconómicos para analizar si el comportamiento de estos países en lo que a educación se refiere va acompañado de algún patrón en el resto de los indicadores analizados.

Palabras clave: Género, educación, Europa, Análisis Factorial Múltiple, contexto socioeconómico.

\section{Education in Europe from a Gender Perspective}

\begin{abstract}
In this paper we analyze education in European countries, both overall and from a gender perspective, and we use the information derived from this analysis to compare the educational situation of these countries with the one that we observe in several indicators which reflect other socioeconomic aspects thereof. For multidimensional analysis of the state of education taking into account gender, we use the Multiple Factor Analysis exploratory technique in order to analyze globally the relative positions of these countries as far as education is concerned. In addition, the educational situation of these countries, summarized by the first factors of the previous analysis, has been combined with the information provided to us by several socioeconomic indicators with the ultimate goal of detecting whether the behaviour of these countries as far as education is concerned is accompanied by some pattern in the rest of the analyzed indicators.
\end{abstract}

Keywords: Gender, Education, Europe, Multiple Factor Analysis, Socioeconomic Context.

Clasificación JEL: C38, I21

*Agradecemos a los dos evaluadores anónimos su contribución a la mejora de este trabajo.

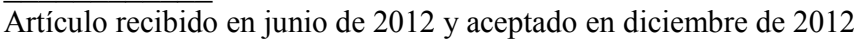

Artículo disponible en versión electrónica en la página www.revista-eea.net, ref. ə-31106 


\section{INTRODUCCIÓN}

La igualdad entre mujeres y hombres es un derecho fundamental y un principio común de la Unión Europea (UE) que, desde su creación, está comprometida con el fomento de la igualdad de género ${ }^{1}$. Prueba de ello es la inclusión en el Tratado de Roma del artículo en el que se establece el principio de que los hombres y las mujeres deben recibir igual salario por un mismo trabajo (Artículo 119 del Tratado de Roma, 1957) (ECT, 1957). Posteriormente, la entrada en vigor del Tratado de Ámsterdam fortaleció el compromiso europeo con la igualdad de género, dado que el mismo especifica como uno de sus objetivos fundamentales la eliminación de las desigualdades y el fomento de la igualdad entre hombres y mujeres en todas las actividades de la UE (CE, 1999). Con la integración de la igualdad de oportunidades, todas las medidas e intervenciones deben tener en cuenta de manera abierta y activa los efectos sobre la situación respectiva de mujeres y hombres (CE, 2002). En la actualidad, la igualdad de oportunidades entre mujeres y hombres sigue siendo uno de los principios rectores de las políticas europeas y del derecho comunitario, formando parte de la Agenda Renovada de Lisboa (CE, 2005) y de la Estrategia Europa 2020 (CE, 2010).

Pese a las importantes consecuencias que las políticas para la igualdad de género pueden tener sobre la educación ${ }^{2}$, en la Unión Europea éstas han estado centradas, principalmente, en la participación de la mujer en el mercado de trabajo, sobre todo en incrementar su participación en Matemáticas, Ciencias y Tecnología (Lynch y Feeley, 2009). A ello ha contribuido, quizás, el hecho de que en la UE la política educativa sea competencia de cada país ${ }^{3}$. No obstante lo anterior, después de décadas de atención a las cuestiones de género en la UE, en lo relativo a educación se han hecho importantes avances. Las mujeres han mejorado bastante su nivel educativo y, en muchos países, han sobrepasado a los

\footnotetext{
${ }^{1}$ La igualdad de género, igualdad entre hombres y mujeres, implica que todos los seres humanos, hombres y mujeres, son libres para desarrollar sus aptitudes personales y tomar sus propias decisiones sin ningún tipo de limitación basada en estereotipos, en una división rígida de los roles de género o en prejuicios. La equidad de género significa que el trato a hombres y mujeres ha de ser imparcial, de acuerdo con sus respectivas necesidades. El trato puede ser el mismo o puede ser diferente, pero ha de ser equivalente en lo que se refiere a derechos, prestaciones, obligaciones y oportunidades (UNESCO, 2000).

${ }^{2}$ En los países europeos, la igualdad de género se contempla en la legislación de diferentes formas y en distinto grado, y se la define de variadas maneras. En la mayor parte de los países europeos se entiende por igualdad de género en el ámbito educativo tanto la igualdad de trato y de oportunidades como la igualdad de resultados (Red Eurydice, 2010).

${ }^{3}$ De acuerdo con lo dispuesto en los artículos 165 y 166 del Tratado de Funcionamiento de la Unión Europea, la UE apoya y completa la acción de los Estados miembros, respetando plenamente la responsabilidad de los mismos en lo relativo al diseño de los contenidos de la enseñanza y a la organización del sistema educativo (UE, 2010).
} 
hombres en cuanto a resultados en exámenes oficiales. Sin embargo, siguen existiendo desigualdades de género, que son especialmente notables en la baja participación de las mujeres en Matemáticas, Ciencias y Tecnología y en los pobres resultados de los hombres (dentro de la clase trabajadora y entre los pertenecientes a algunas minorías) en cuanto a nivel de formación (Lynch y Feeley, 2009).

En este contexto, el objetivo principal de este trabajo es analizar, desde la perspectiva de género, la situación actual de un grupo de países europeos en cuanto a los resultados de la educación y la formación y su conexión con el contexto socioeconómico de los mismos. Dicho análisis se lleva a cabo en varias fases. En la primera de ellas, describimos, teniendo en cuenta el género, el comportamiento unidimensional de los distintos indicadores educativos en los países a los que el estudio se refiere. En la segunda fase utilizamos la técnica estadística multidimensional de Análisis Factorial Múltiple (AFM) para analizar, de manera global y atendiendo al género, la situación educativa de los mismos. Por último, complementamos el análisis previo sobre el estado de la educación en Europa con la información que nos proporcionan diversos indicadores socioeconómicos, planteándonos un doble objetivo. Por una parte, tratamos de establecer si existe relación entre la situación de estos países con respecto a las características socioeconómicas consideradas y su situación educativa, resumida esta última por los primeros factores del análisis factorial. Por otra parte, y centrándonos en las diferencias de género, analizamos cómo de acentuadas son las diferencias entre hombres y mujeres en relación a las otras características socioeconómicas en aquellos países que presentan las mayores diferencias en cuanto a educación.

El trabajo está estructurado del siguiente modo: en la Sección 2 hacemos referencia a los países objeto de estudio, definimos los indicadores utilizados para caracterizar la situación educativa de los mismos y llevamos a cabo su descripción unidimensional; en la Sección 3 describimos, brevemente, la técnica estadística de Análisis Factorial Múltiple que utilizamos para el análisis multidimensional de la educación y presentamos los resultados de dicho análisis para los indicadores educativos; en la Sección 4 definimos los indicadores socioeconómicos considerados y realizamos un análisis conjunto de los mismos y la situación educativa, resumida por los primeros factores obtenidos en el análisis previo, utilizando la técnica estadística de Análisis de Conglomerados; y, por último, en la Sección 5 presentamos las conclusiones de este estudio. 


\section{INDICADORES EDUCATIVOS: DEFINICIONES Y ANÁLISIS UNIDIMENSIONAL}

Este estudio está referido a veinticuatro países de la Unión Europea ${ }^{4}$, cuatro países candidatos (Croacia, Islandia, Macedonia y Turquía) y otros dos países europeos (Noruega y Suiza). Los indicadores estadísticos que utilizamos para conseguir el objetivo propuesto proceden de la Oficina de Estadística de la Unión Europea (Eurostat). Estos indicadores se han dividido en dos grupos ${ }^{5}$ :

Grupo 1: Indicadores de la Unión Europea (UE) que son utilizados como punto de referencia general de la Estrategia 2020 y de los Objetivos 2010 y 2020 de los sistemas educativos y formativos en la UE (C, 2009; CE, 2007 y 2010).

Grupo 2: Otros indicadores internacionales de educación elaborados por Eurostat y la OCDE (Indicadores de la Educación de la OCDE, Proyecto INES).

En la Tabla 1 se presentan las definiciones de los distintos indicadores utilizados, junto con el grupo en el que se enmarcan y el año al que corresponden los datos. El apartado que sigue está dedicado al análisis descriptivo de dichos indicadores.

\subsection{Descripción unidimensional de las características educativas desde la perspectiva de género}

Comenzamos el análisis con las variables relativas a la población joven (AET, FES, FPJ, NJ1, NJ3, T15 y T16). En general, destacamos que, por término medio, en la UE el nivel de formación de los hombres jóvenes es un poco inferior al de las mujeres de su misma edad. En 2010 un $37,5 \%$ de las mujeres entre 25 y 34 años había alcanzado el nivel de formación en Educación Superior (NJ3), mientras que este porcentaje ascendió sólo a un $28,9 \%$ entre los hombres ${ }^{6}$. En todos los países, salvo en Suiza y Turquía, la diferencia es a favor de las mujeres, siendo las más acentuadas, entre 21,6 y 15,4 puntos porcentuales, las correspondientes a Letonia, Eslovenia, Lituania, Finlandia y Noruega.

\footnotetext{
${ }^{4}$ Se han excluido Estonia, Grecia y Luxemburgo por no disponer de datos para algunas de las variables que se consideran relevantes para este estudio.

${ }^{5}$ La selección de las variables o indicadores que utilizamos en este estudio queda condicionada, como en la mayor parte de los trabajos de índole práctico, por la información útil disponible en el medio que utilizamos como fuente de información, Eurostat.

${ }^{6} \mathrm{Si}$ nos restringimos a los jóvenes con edad entre 30 y 34 años, los porcentajes de población con nivel de formación en educación superior (FES) son de un 37,2 \% entre las mujeres frente a un $30 \%$ entre los hombres.
} 
Tabla 1

Definiciones de los indicadores de educación

\begin{tabular}{|c|c|c|}
\hline Variable & Símbolo & Definición y año \\
\hline $\begin{array}{l}\text { Abandono Educativo } \\
\text { Temprano }\end{array}$ & AET & $\begin{array}{l}\text { Porcentaje de la población entre } 18 \text { y } 24 \text { años que posee un } \\
\text { nivel de educación inferior a Educación Secundaria } 2^{\mathrm{a}} \text { etapa y no } \\
\text { sigue ningún programa de estudio o formación (Grupo 1), } 2010 \text {. }\end{array}$ \\
\hline $\begin{array}{l}\text { Formación en Educación } \\
\text { Superior en la Población } \\
\text { de } 30 \text { a } 34 \text { años }\end{array}$ & FES & $\begin{array}{l}\text { Porcentaje de población entre } 30 \text { y } 34 \text { años que ha alcanzado el } \\
\text { nivel de formación en Educación Superior (Grupo 1), } 2010 .\end{array}$ \\
\hline Formación Permanente & FP & $\begin{array}{l}\text { Porcentaje de población entre } 25 \text { y } 64 \text { años que participa en } \\
\text { educación y formación (Grupo 1), } 2010 .\end{array}$ \\
\hline $\begin{array}{l}\text { Formación de la Población } \\
\text { Joven }\end{array}$ & FPJ & $\begin{array}{l}\text { Porcentaje de población entre } 20 \text { y } 24 \text { años que ha completado } \\
\text { el nivel de Educación Secundaria } 2^{\mathrm{a}} \text { etapa (Grupo 1), } 2010 .\end{array}$ \\
\hline $\begin{array}{l}\text { Tasa de Participación en } \\
\text { Matemáticas, Ciencias y } \\
\text { Tecnología }\end{array}$ & МСТЕ & $\begin{array}{l}\text { Porcentaje de matriculados en estudios superiores de } \\
\text { Matemáticas, Ciencias y Tecnología con respecto al total de } \\
\text { estudiantes universitarios (Grupo 1), 2009. }\end{array}$ \\
\hline $\begin{array}{l}\text { Tasa de Graduación en } \\
\text { Matemáticas, Ciencias y } \\
\text { Tecnología }\end{array}$ & MCTG & $\begin{array}{l}\text { Porcentaje de graduados en estudios superiores de } \\
\text { Matemáticas, Ciencias y Tecnología con respecto al total de } \\
\text { graduados universitarios (Grupo 1), } 2009 \text {. }\end{array}$ \\
\hline $\begin{array}{l}\text { Nivel de Formación de la } \\
\text { Población Adulta-1 }\end{array}$ & NA1 & $\begin{array}{l}\text { Porcentaje de la población de } 25 \text { a } 64 \text { años con nivel inferior a } \\
\text { Educación Secundaria } 2^{\mathrm{a}} \text { etapa (Grupo } 2 \text { ), } 2010 .\end{array}$ \\
\hline $\begin{array}{l}\text { Nivel de Formación de la } \\
\text { Población Adulta-3 }\end{array}$ & NA3 & $\begin{array}{l}\text { Porcentaje de la población de } 25 \text { a } 64 \text { años con Educación } \\
\text { Superior (Grupo 2), } 2010 .\end{array}$ \\
\hline $\begin{array}{l}\text { Nivel de Formación de la } \\
\text { Población Joven-1 }\end{array}$ & NJ1 & $\begin{array}{l}\text { Porcentaje de la población de } 25 \text { a } 34 \text { años con nivel inferior a } \\
\text { Educación Secundaria } 2^{\mathrm{a}} \text { etapa (Grupo } 2 \text { ), } 2010 .\end{array}$ \\
\hline $\begin{array}{l}\text { Nivel de Formación de la } \\
\text { Población Joven-3 }\end{array}$ & NJ3 & $\begin{array}{l}\text { Porcentaje de la población de } 25 \text { a } 34 \text { años con Educación } \\
\text { Superior (Grupo 2), } 2010 .\end{array}$ \\
\hline $\begin{array}{l}\text { Porcentaje de Empleados } \\
\text { con Bajo Nivel Educativo }\end{array}$ & PEB & $\begin{array}{l}\text { Porcentaje del total de empleados que representan aquéllos con } \\
\text { nivel educativo inferior a la Educación Secundaria } 2^{\mathrm{a}} \text { etapa } \\
\text { (Grupo 2), 2010. }\end{array}$ \\
\hline $\begin{array}{l}\text { Porcentaje de Empleados } \\
\text { con Alto Nivel Educativo }\end{array}$ & PES & $\begin{array}{l}\text { Porcentaje del total de empleados que representan aquéllos con } \\
\text { Educación Superior (Grupo 2), } 2010 .\end{array}$ \\
\hline $\begin{array}{l}\text { Tasa de Participación en } \\
\text { Educación en la Edad de } \\
15 \text { a } 24 \text { años }\end{array}$ & T15 & $\begin{array}{l}\text { Razón entre el alumnado con edad entre } 15 \text { y } 24 \text { años que está } \\
\text { escolarizado en cualquier nivel educativo y el total de población } \\
\text { de la misma edad (Grupo 2), } 2009 \text {. }\end{array}$ \\
\hline $\begin{array}{l}\text { Tasa de Participación en } \\
\text { Educación en la Edad de } \\
16 \text { a } 18 \text { años }\end{array}$ & T16 & $\begin{array}{l}\text { Razón entre el alumnado con edad entre } 16 \text { y } 18 \text { años que está } \\
\text { escolarizado en cualquier nivel educativo y el total de población } \\
\text { de la misma edad (Grupo } 2 \text { ), } 2009 \text {. }\end{array}$ \\
\hline
\end{tabular}

Fuente: Eurostat.

Paralelamente, en este mismo tramo de edad, un 21\% de los hombres tiene un nivel de formación inferior a la Educación Secundaria $2^{\mathrm{a}}$ etapa $(\mathrm{NJ} 1)$, frente a un $17,5 \%$ de las mujeres (valores medios UE-27). Las diferencias más acentuadas a favor de los hombres, entre 14 y 6,5 puntos porcentuales, las presentan Portugal, España, Islandia, Letonia e Italia, mientras que las más acentuadas a favor de las mujeres las observamos en Turquía y Macedonia, 13 y 9,5 puntos porcentuales, respectivamente.

En contrapartida, los porcentajes de hombres o mujeres que han completado el nivel de Educación Secundaria $2^{\mathrm{a}}$ etapa (FPJ) son muy similares entre las personas con edad entre 20 y 24 años, 64,9\% entre los hombres frente a 64,7\% 
entre las mujeres. Dinamarca, Noruega, Malta e Italia presentan las diferencias más acentuadas a favor de las mujeres, entre 11,5 y 4 puntos porcentuales; sin embargo, en Chipre, Turquía y Macedonia observamos diferencias bastante elevadas a favor de los hombres, entre 27,6 y 11 puntos porcentuales.

En cuanto al porcentaje de la población entre 18 y 24 años que posee un nivel de educación inferior a Educación Secundaria $2^{\mathrm{a}}$ etapa y no sigue ningún programa de educación o formación (AET), el valor medio (UE-27) para los hombres se sitúa en un $16 \%$, mientras que entre las mujeres es de un $12,2 \%$. En la mayor parte de los países, a excepción de Turquía, Macedonia, Bulgaria, Suiza y Eslovaquia, los valores correspondientes a las mujeres para este indicador son inferiores a los que presentan los hombres. Las diferencias más acusadas en este sentido (entre 10,4 y 7 puntos porcentuales) las observamos en España, Malta, Noruega, Portugal, Letonia e Islandia.

En lo relativo a la participación en educación de la población joven (T15 y $\mathrm{T} 16)^{7}$, también fue ligeramente superior la correspondiente a la mujer, tanto entre 16 y 18 años como entre 15 y 24 . Por término medio, un $88,3 \%$ de las mujeres con edad entre 16 y 18 años y un $62,4 \%$ de las que tienen entre 15 y 24 años participa en educación, ascendiendo estos porcentajes entre los hombres a un $85,3 \%$ y un $57,9 \%$, respectivamente. En la mayor parte de los países analizados las diferencias en participación son a favor de las mujeres. En Eslovenia, Letonia, Rumanía, Croacia, Eslovaquia y Suecia observamos las diferencias más acentuadas en cuanto a participación a la edad de 15 a 24 años, mientras que, a la edad de 16 a 18 años las diferencias en participación más acentuadas las observamos en Chipre, España, Reino Unido, Croacia, Irlanda y Portugal.

Centrándonos en la población adulta (entre 25 y 64 años), aunque el porcentaje de mujeres con nivel inferior a Educación Secundaria $2^{\mathrm{a}}$ etapa (NA1) es ligeramente superior al de hombres, $28,3 \%$ frente a $26,3 \%$ (valores medios para mujeres y hombres en EU-27), el porcentaje de mujeres en ese tramo de edad con formación superior (NA3) excede ligeramente al de los hombres $(26,7$ $\%$ frente a $25,1 \%$ ) y también es mayor su participación en educación y formación (FP), un $10 \%$ de las mujeres frente al 8,3\% de los hombres. En cuanto a los países en los que se observan las diferencias más acentuadas entre mujeres y hombres en este grupo de variables, destacamos Letonia, Lituania, Finlandia, Islandia, Suecia y Bulgaria, con diferencias a favor de las mujeres de más de 10 puntos porcentuales en el indicador que cuantifica la formación en educación superior (NA3). En Suecia y Dinamarca se observan diferencias de más de 13 puntos porcentuales a favor de las mujeres en cuanto a participación en educación y formación (FP) y en Macedonia, Turquía y Austria observamos que el

\footnotetext{
${ }^{7}$ Los datos relativos a participación corresponden al año 2009, último para el que hay información sobre esta variable en Eurostat.
} 
porcentaje de mujeres con educación inferior a la Educación Secundaria $2^{\mathrm{a}}$ etapa es superior en más de 10 puntos porcentuales al de hombres.

En cuanto a las tasas de participación y graduación en Matemáticas, Ciencias y Tecnología (MCTE y MCTG), en todos los países analizados los valores de estos indicadores entre los hombres son muy superiores a los que se observan entre las mujeres. Las diferencias más acentuadas en cuanto a participación las encontramos en Finlandia, Lituania, Eslovenia, Alemania y República Checa, entre 41,6 y 29,2 puntos porcentuales. En Finlandia, Lituania, Austria, Suecia, República Checa y España observamos las mayores diferencias en lo que a graduación se refiere, entre 40,4 y 29,3 puntos porcentuales.

Por último, en relación al nivel de formación de las personas empleadas (PEB y PES), el 22,1 \% de los hombres empleados y el 18,9\% de las mujeres tenían en 2010 un nivel de formación inferior a la Educación Secundaria $2^{\mathrm{a}}$ etapa. Asimismo, un $27 \%$ de los hombres empleados y un 32,5\% de las mujeres tenían el nivel más alto de educación (valores medios UE-27). En relación a este último indicador en la mayor parte de los países considerados el valor del mismo en las mujeres es superior al del hombre (sólo en tres de los treinta que entran en el análisis ocurre lo contrario) y las diferencias más acentuadas, más de 15 puntos porcentuales, las observamos en Turquía, Lituania, Polonia, Letonia y Bulgaria.

\section{ANÁLISIS MULTIDIMENSIONAL DE LA EDUCIÓN EN EUROPA}

Como ya hemos comentado, para el análisis de la educación desde una perspectiva multidimensional que tenga en cuenta los valores de los indicadores desglosados por género hemos aplicado la técnica estadística exploratoria denominada Análisis Factorial Múltiple.

El Análisis Factorial Múltiple (AFM) es una técnica exploratoria para el análisis de datos multidimensionales relativos a un conjunto de elementos que son descritos mediante varios grupos de variables (Escofier y Pagès, 1990, 1992 y 1994). Los grupos pueden diferir en número de variables y en la naturaleza de las mismas (cuantitativas o cualitativas), pero dentro de cada grupo todas las variables deben ser del mismo tipo (Pagès, 2004). En lo que sigue nos referiremos a su aplicación a grupos formados por variables cuantitativas, teniendo en cuenta que no está entre los objetivos de este trabajo el realizar un análisis detallado de los fundamentos teóricos del AFM. En Escofier y Pagès (1990) y en Lebart, Morineau y Piron (1995) se puede encontrar una presentación completa de este método.

El punto de partida para la aplicación del AFM es una tabla que contiene tantas filas como unidades de análisis y cuyas columnas están formadas por los valores que en dichas unidades toman las variables que conforman los distintos 
grupos o bloques. La implementación del AFM se lleva a cabo en dos etapas sucesivas: en la primera se realizan análisis separados de las matrices de correlación correspondientes a los distintos grupos de variables mediante la técnica de Análisis de Componentes Principales (ACP) y se selecciona el primer autovalor de cada uno de esos análisis parciales; en la segunda etapa se aplica ACP a la matriz formada por todos los grupos de variables. Este análisis, en adelante análisis global, está basado en la matriz de covarianzas correspondiente a las variables de todos los grupos tipificadas y ponderadas. Todas las variables de un grupo se tipifican con respecto al mismo y se ponderan con el inverso de la raíz del primer autovalor del ACP de dicho grupo. De este modo, la inercia de la primera componente principal de cada grupo de variables es igual a uno y la suma de las inercias de las variables de un mismo grupo sobre un eje cualquiera del espacio es inferior o igual a uno, equilibrando así la contribución de cada uno de los grupos en la formación del primer factor del AFM y conservando la estructura interna de cada grupo (Escofier y Pagès, 1990).

Como se indica en Abascal y otros (2001), el AFM proporciona indicadores y gráficos que son semejantes a los del resto de análisis factoriales y otros específicos que permiten: por un lado, el estudio de las relaciones entre los grupos y medir el grado de semejanza global entre los mismos; por otro lado, el análisis de las relaciones entre las variables de un grupo y las del resto de los grupos y, por último, las semejanzas entre los individuos vistos a través de los diferentes grupos de variables y en conjunto.

Para analizar la semejanza global entre los distintos grupos considerados (inter-estructura) se utilizan, básicamente: las matrices de coeficientes Lg y RV, las coordenadas de los grupos sobre los factores del análisis global y las correlaciones entre los factores del análisis global y sus representantes en los grupos, es decir, los factores de los análisis parciales (García y Abascal, 2003).

Por su parte, el coeficiente RV (Escoufier, 1973) es una medida del grado de relación entre dos grupos de variables y está basado en la idea de que dichos grupos están perfectamente correlacionados si existe una transformación ortogonal que hace que los dos grupos coincidan (Josse y otros, 2008). El valor de este coeficiente, que está comprendido entre cero (ninguna de las variables del grupo X está correlacionada con las variables del grupo Y) y uno (las nubes que representan a dichos grupos son homotéticas), se puede interpretar como un coeficiente de correlación entre los dos grupos de variables X e Y.

La información que proporciona la matriz de coeficientes RV se completa con la dada por la matriz de coeficientes Lg, también basados en los RV. El valor del coeficiente $\mathrm{Lg}$ es cero cuando las correlaciones entre cada variable de $\mathrm{X}$ y cualquiera de $\mathrm{Y}$ son todas nulas. Por el contrario, el valor mayor se alcanzará cuando la relación lineal entre cada una de las variables de $\mathrm{X}$ y cualquiera de las de Y sea perfecta. Aunque dicho valor máximo depende del número de 
variables en cada uno de los grupos, valores altos de este indicador reflejan que $\mathrm{X}$ e Y son multidimensionales y presentan factores comunes que están próximos a los factores parciales de dichos grupos (Pagès, 1998).

El análisis de los coeficientes de correlación entre los factores de los análisis parciales y los factores del análisis global permite poner en evidencia cuáles son los factores comunes a todos los grupos, los comunes sólo a algunos grupos y los específicos de un grupo, en la medida en que dichos coeficientes (que son siempre positivos y se denominan coeficientes de correlación canónica) sean próximos a uno en todos los grupos, sólo en algunos o sólo en uno (Pagès, 2004). Por último, se utilizan las coordenadas de los distintos grupos sobre los factores globales, teniendo en cuenta que, la coordenada de un grupo sobre un factor global representa la relación entre las variables englobadas en el mismo y dicho factor. Esta información se complementa con la proporcionada por las contribuciones de cada grupo a la formación del factor.

Por otra parte, el estudio de la intra-estructura o compromiso permite detectar el número de factores comunes a los distintos grupos. Para este objetivo el AFM proporciona como principal indicador las inercias intra ${ }^{8}$ de los puntos correspondientes a los individuos. Así, los individuos con puntos parciales próximos (inercia intra débil) ilustran la estructura común de las distintas tablas analizadas. Por el contrario, los que tienen asociados puntos parciales que están alejados entre sí (inercia intra alta), constituyen las excepciones a la estructura común (Abascal y Landaluce, 2002). Las representaciones gráficas de individuos y variables en los planos factoriales asociados al análisis global, que se interpretan como las correspondientes a un ACP (las coordenadas de un individuo en un plano son sus puntuaciones en los factores que lo forman y las coordenadas de una variable son sus correlaciones con dichos factores), permiten observar la proximidad entre los distintos puntos que representan a un mismo individuo y dotar a los ejes factoriales de un significado.

\subsection{Resultados del Análisis Factorial Múltiple aplicado a las características educativas}

El punto de partida en la aplicación de esta técnica es la matriz de datos formada por los valores, en los 30 países analizados, de dos grupos de catorce variables que representan tanto la participación en educación y formación como el nivel de formación en distintos tramos de edad para hombres (Grupo 1) y muje-

\footnotetext{
${ }^{8}$ La inercia total, o variabilidad de la nube global (es decir, individuos teniendo en cuenta todas las variables) respecto de su centro de gravedad, se descompone de manera aditiva en inercia intra e inercia inter. La inercia intra es la inercia de los puntos parciales (individuos caracterizados por un grupo de variables) con respecto a su punto medio en la nube global. La inercia inter es la inercia de los centros de gravedad de cada nube parcial con respecto al centro de gravedad de la nube global (Abascal y otros, 2006).
} 
res (Grupo 2). El objetivo de la aplicación de esta técnica es, como ya hemos comentado, analizar de manera global las posiciones relativas de los países considerados en lo que a educación y formación se refiere, poniendo de manifiesto: las diferencias y similitudes entre las estructuras de ambos grupos, además de cuantificar su grado de semejanza; la existencia de factores comunes a hombres y mujeres y de otros específicos de alguno de ellos, así como, las semejanzas y diferencias entre países y variables, tanto globalmente como por género.

Como paso previo, necesario para la implementación del AFM, hemos realizado los ACP correspondientes a los grupos considerados, hombres y mujeres. En la Tabla 2 se muestran los primeros autovalores de dichos análisis. Siguiendo el criterio de retener las componentes asociadas a autovalores superiores a la unidad (Kaiser, 1960), observamos que el número de factores necesarios para describir la situación en educación y formación de estos países no es el mismo en ambos grupos, resultando tres factores para las variables relativas a la participación en educación y al nivel de formación de los hombres frente a cuatro para la estructura relativa a las mujeres. Ello puede ser un primer indicio de que, posiblemente, los grupos analizados, además de compartir una estructura común, presenten rasgos específicos.

Tabla 2

Primeros autovalores de los ACP

\begin{tabular}{|c|c|c|c|c|c|}
\hline \multicolumn{3}{|c|}{ Hombres (Grupo 1) } & \multicolumn{3}{c|}{ Mujeres (Grupo 2) } \\
\hline Autovalores & \% de varianza & \% acumulado & Autovalores & \% de varianza & \% acumulado \\
\hline 6,3064 & 45,0454 & 45,0454 & 6,8041 & 48,6007 & 48,6007 \\
\hline 3,9439 & 28,1706 & 73,2161 & 3,2606 & 23,2901 & 71,8907 \\
\hline 1,7035 & 12,1677 & 85,3837 & 1,5939 & 11,3847 & 83,2755 \\
\hline 0,9204 & 6,5741 & 91,9579 & 1,0361 & 7,4009 & 90,6764 \\
\hline 0,6127 & 4,3761 & 96,3340 & 0,6285 & 4,4890 & 95,1654 \\
\hline
\end{tabular}

Fuente: Elaboración propia.

La matriz global, que es el punto de partida en el desarrollo del $\mathrm{AFM}^{9}$, resulta al yuxtaponer las matrices formadas por los indicadores iniciales tipificados y ponderados correspondientes a estos grupos. Los indicadores de cada grupo se ponderan con el inverso de la raíz del primer autovalor que resulta de su correspondiente ACP. La aplicación de un ACP a la matriz de covarianzas asociada a la global es la etapa principal del AFM. Los primeros autovalores del análisis global (AFM) junto con el porcentaje de varianza que explican los fac-

\footnotetext{
${ }^{9}$ Para el análisis de la matriz global mediante AFM se ha utilizado el software SPAD 5.6 (Systéme Pour L'Analyse des Données).
} 
tores asociados a los mismos (en adelante, factores "globales" para diferenciarlos de los factores "parciales" que se obtienen en los ACP de los grupos) se muestran en la Tabla 3. Asimismo, en la Figura 1 presentamos éstos junto a los correspondientes a los análisis separados de ambos grupos.

Tabla 3

Primeros autovalores del AFM

\begin{tabular}{|c|c|c|}
\hline Autovalores & \% de varianza & \% acumulado \\
\hline 1,9140 & 44,7451 & 44,7451 \\
1,0608 & 24,7983 & 69,5434 \\
0,4230 & 9,8877 & 79,4311 \\
0,3017 & 7,0541 & 86,4852 \\
0,2494 & 5,8300 & 92,3151 \\
0,0830 & 1,9408 & 94,2560 \\
\hline
\end{tabular}

Fuente: Elaboración propia.

Figura 1

Primeros autovalores de los ACP por grupos y del AFM

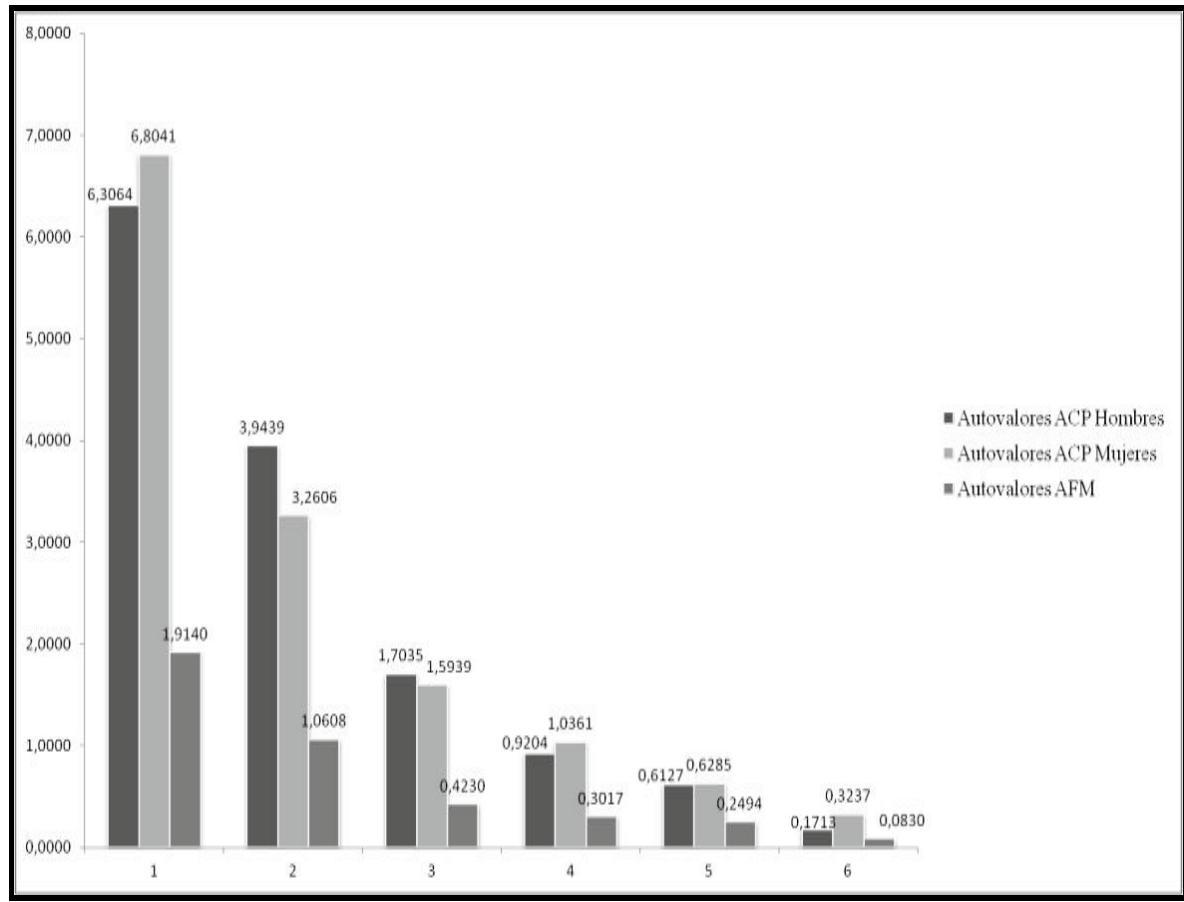

Fuente: Elaboración propia. 
La proximidad del primer valor propio del AFM $(1,91)$ al número de grupos considerados, que sería su máximo posible, y el alto porcentaje de la variabilidad total que explica el factor asociado al mismo (casi un 45\%) son los primeros indicios de que dicho factor es común a los dos grupos. Por otra parte, siguiendo el mismo criterio que para los ACP parciales en cuanto al número de factores a retener y teniendo en cuenta que, al trabajar con las variables ponderadas, en el AFM la variabilidad media es de 0,15, sería conveniente, si admiten una interpretación clara en el contexto analizado, retener los cinco primeros factores globales que, en conjunto, permitirían explicar más del 92\% de la variabilidad total.

\subsubsection{Análisis de la inter-estructura}

En este punto analizamos la proximidad entre las matrices correspondientes a los dos grupos. En las Tablas 4 y 5 se muestran las matrices de coeficientes $\mathrm{Lg}$ y RV, respectivamente. Estas matrices nos proporcionan información sobre la dimensionalidad de los dos grupos de variables y la relación entre ellos. Los valores de la diagonal principal de la matriz de coeficientes Lg indican que los dos grupos y el global presentan una estructura similar en cuanto al número de factores con inercia considerable.

Adicionalmente, la matriz de coeficientes RV proporciona información directa sobre el grado de relación entre los dos grupos y entre éstos y el global. De la observación de los valores de estos coeficientes (Tabla 5) concluimos que la relación entre el grupo de variables que describe la situación en educación y formación de los hombres y el correspondiente a las mujeres es bastante acentuada $(0,83)$, pero lo es bastante más la correspondiente a cada uno de esos grupos con el global (superior a 0,95 en ambos casos).

Tabla 4

Coeficientes $\mathrm{Lg}$ de relación entre grupos

\begin{tabular}{|l|c|c|c|}
\hline & Hombres & Mujeres & AFM \\
\hline Hombres & 1,4962 & & \\
Mujeres & 1,1612 & 1,3191 & \\
AFM & 1,3884 & 1,2959 & 1,4024 \\
\hline
\end{tabular}

Fuente: Elaboración propia
Tabla 5

Coeficientes $\mathrm{RV}$ de relación entre grupos

\begin{tabular}{|l|c|c|c|}
\hline & Hombres & Mujeres & AFM \\
\hline Hombres & 1,0000 & & \\
Mujeres & 0,8265 & 1,0000 & \\
AFM & 0,9585 & 0,9527 & 1,0000 \\
\hline
\end{tabular}

Fuente: Elaboración propia.

El análisis de los coeficientes de correlación entre los factores del análisis global y las variables representantes de los grupos en los análisis parciales (Tabla 6) pone en evidencia que los tres primeros factores del análisis global, con correlaciones mayores o iguales que 0,9 , reflejan aspectos que son comunes a 
ambos grupos, siendo la correlación ligeramente más alta con las variables representantes del grupo de los hombres.

Tabla 6

Correlaciones entre variables canónicas y factores del análisis global

\begin{tabular}{|l|c|c|c|c|c|}
\hline \multirow{2}{*}{ Grupo } & & \multicolumn{4}{|c|}{ Correlaciones } \\
\cline { 2 - 6 } & Factor 1 & Factor 2 & Factor 3 & Factor 4 & Factor 5 \\
\hline Hombres & 0,9805 & 0,9809 & 0,9240 & 0,8089 & 0,6976 \\
Mujeres & 0,9804 & 0,9676 & 0,8936 & 0,7584 & 0,7874 \\
\hline
\end{tabular}

Fuente: Elaboración propia.

Los factores cuatro y cinco, cuya correlación con las variables representantes de los grupos es un poco menor (entre 0,7 y 0,8 ), muestran diferencias más acentuadas en cuanto a su relación con los grupos. En este sentido, es más alta la correlación entre el factor global cuatro y las variables representantes del grupo de los hombres y para el factor cinco es más alta con las correspondientes a las mujeres.

Las coordenadas de los distintos grupos sobre los factores globales y sus contribuciones a la formación de los mismos (Tabla 7) dejan patente que la relación existente entre los factores del análisis global y el grupo de variables relativas a los hombres es, en general, más alta y que dicho grupo contribuye en mayor medida a la formación de los ejes globales.

Tabla 7

Coordenadas y ayuda a la interpretación de los grupos activos

\begin{tabular}{|c|c|c|c|c|c|c|c|c|c|c|}
\hline \multirow{2}{*}{ Grupo } & \multicolumn{5}{|c|}{ Coordenadas } & \multicolumn{5}{|c|}{ Contribuciones } \\
\hline & F1 & F2 & F3 & F4 & F5 & F1 & F2 & F3 & F4 & F5 \\
\hline Hombres & 0,96 & 0,60 & 0,23 & 0,16 & 0,11 & 50,12 & 56,83 & 54,83 & 54,18 & 43,25 \\
\hline Mujeres & 0,95 & 0,46 & 0,19 & 0,14 & 0,14 & 49,88 & 43,17 & 45,17 & 45,82 & 56,75 \\
\hline & & & & & & 1,00 & 1,00 & 1,00 & 1,00 & 1,00 \\
\hline
\end{tabular}

Fuente: Elaboración propia.

Las razones entre inercia inter ${ }^{10}$ e inercia total (Tabla 8) reflejan el interés de la representación simultánea de los dos grupos de variables en los tres primeros

${ }^{10}$ Cuando los diferentes conjuntos de variables inducen estructuras similares sobre los individuos, los puntos parciales están próximos entre sí. Esta propiedad global se mide, para cada factor, mediante la razón entre inercia inter e inercia total. Este índice, que varía entre cero y uno, toma un valor próximo a uno cuando el factor representa una estructura que es común a los dos grupos (Pagès, 2004). 
ejes factoriales, idea ya apuntada por las correlaciones entre variables canónicas de los grupos y factores del análisis global (Tabla 6).

Tabla 8

Razón inercia inter-inercia total

\begin{tabular}{|c|c|c|c|c|}
\hline Factor $\mathbf{1}$ & Factor $\mathbf{2}$ & Factor $\mathbf{3}$ & Factor $\mathbf{4}$ & Factor $\mathbf{5}$ \\
\hline 0,96 & 0,94 & 0,82 & 0,61 & 0,55 \\
\hline
\end{tabular}

Fuente: Elaboración propia.

Los resultados comentados hasta este punto reflejan la existencia de una estructura común a ambos grupos que queda recogida en los tres primeros factores globales del AFM. Dichos factores explican casi un $80 \%$ de la variabilidad total.

\subsubsection{Análisis de la intra-estructura}

La representación gráfica de las variables en los primeros planos factoriales, que permite la visualización rápida de cuáles son las que presentan mayor carga en cada factor y cuál es su signo, facilita la interpretación de los mismos. En las Figuras 2 y 3 mostramos las correlaciones entre las variables y los factores en los planos factoriales 1-2 y 1-3, respectivamente. De su análisis podemos concluir que, en general, los dos grupos (variables relativas a hombres y a mujeres) influyen de manera equilibrada en la formación de estos factores; es decir, las variables con correlaciones elevadas con los factores retenidos en el grupo de hombres y en el de mujeres son las mismas. Por ello, en el comentario que sigue, no diferenciaremos las variables por grupo. El primer factor global (F1) representa la situación general en educación y formación de los países analizados $\mathrm{y}$, dado que presenta correlaciones positivas elevadas con variables que muestran aspectos negativos de la educación (PEB, AET, NA1 y NJ1) y correlaciones negativas, también elevadas, con las variables FES, NJ3, PES, NA3, T15 y T16, que representan tanto el nivel de formación en educación superior como la participación en educación de los jóvenes en edades en que ésta no es obligatoria, los valores positivos y altos de este factor irán asociados a países con déficit de formación. Análogamente, valores negativos elevados indicarán países cuya población tiene un nivel educativo elevado y en los que los jóvenes tienen una participación elevada en educación. A este factor lo denominamos "Situación general en educación y formación". 
Figura 2

Variables en el plano factorial 1-2

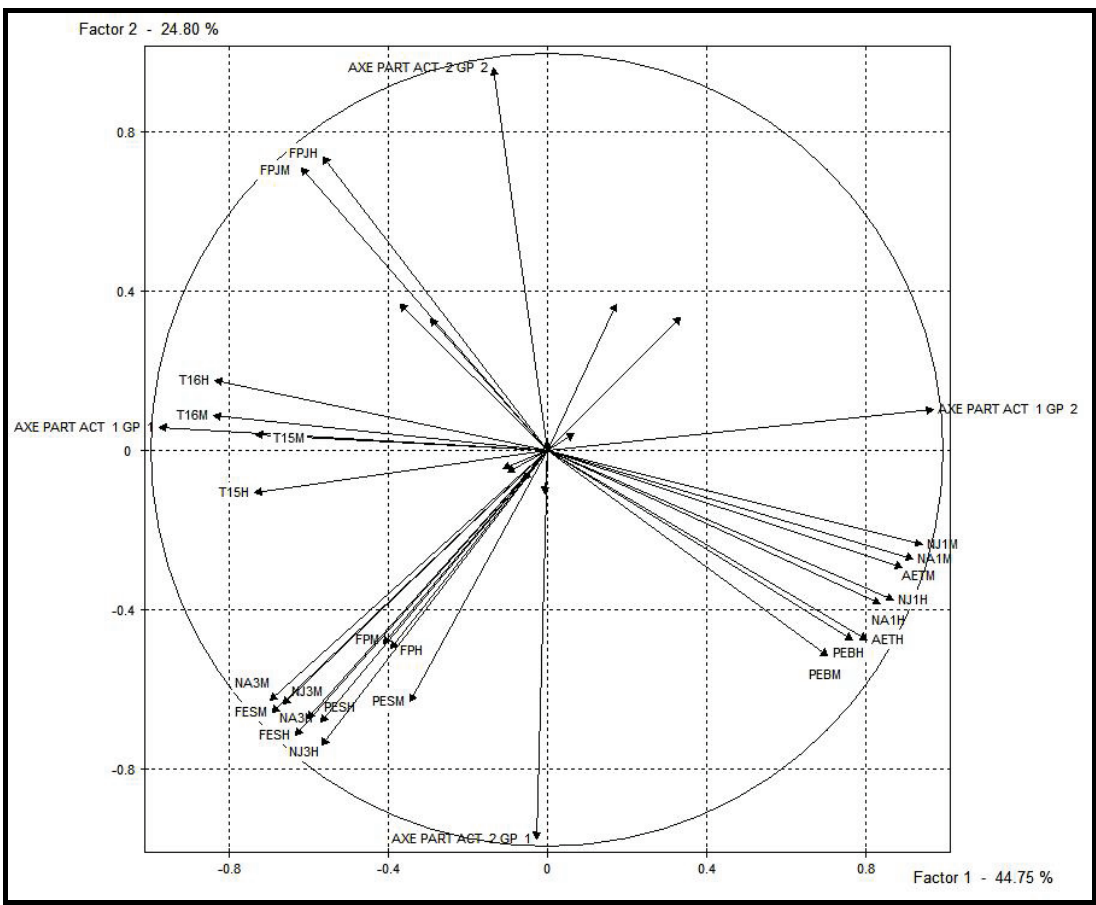

Fuente: Elaboración propia.

En la Figura 2 observamos, también, que las variables que presentan correlaciones elevadas con el segundo factor global (F2) son todas las que reflejan el nivel de formación en educación superior (NA3, NJ3, FES y PES) y la que representa el porcentaje de jóvenes que han completado el nivel de Educación Secundaria $2^{\mathrm{a}}$ etapa (FPJ), siendo con las primeras correlaciones negativas y con esta última positiva, lo que puede dificultar la interpretación de las puntuaciones de los países en el mismo, ya que también presenta correlación negativa, aunque bastante más débil, con la variable que cuantifica la formación permanente (FP). A este factor, que explica casi un $25 \%$ de la variabilidad total, lo denominamos "Formación en Educación Superior", por entender que el peso de las variables que cuantifican este aspecto (cuatro por cada grupo con correlaciones muy elevadas debe compensar con creces al que corresponde a la variable que presenta correlación positiva). No obstante lo anterior, a la hora de interpretar las puntuaciones de países concretos en este factor será necesario recurrir, además, a los datos originales de las variables que cargan sobre él. 
Figura 3

Variables en el plano factorial 1-3

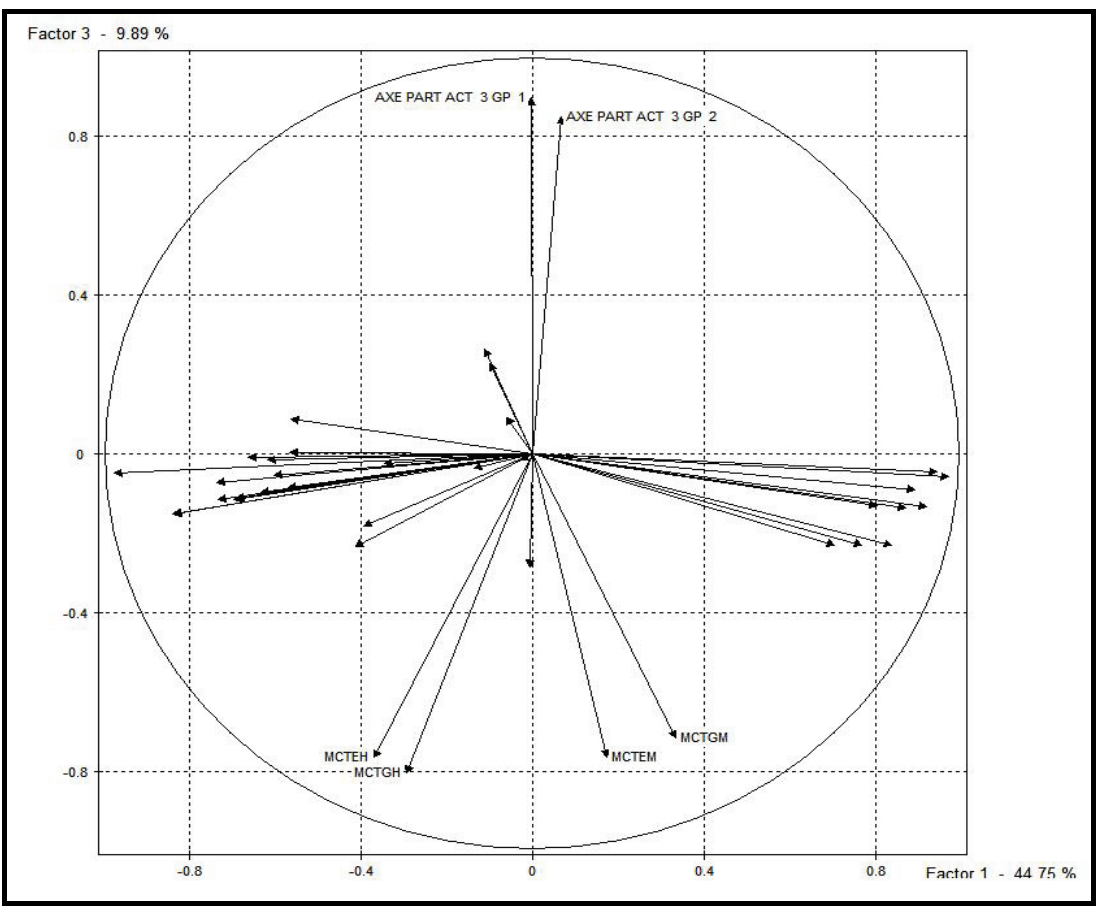

Fuente: Elaboración propia.

En cuanto al tercer factor global (F3), como queda patente en la Figura 3, básicamente representa la participación y graduación en Matemáticas, Ciencias y Tecnología (MCT), por lo que lo denominamos "Participación y graduación en MCT". Este factor explica un $10 \%$ de la variabilidad total y para su interpretación habrá que tener en cuenta que las variables a las que representa están correlacionadas negativamente con él. La participación en educación en el tramo de edad de 15 a 24 años queda recogida en el factor cuatro del análisis global (F4), que presenta correlación elevada sólo con esta variable, siendo la misma positiva. A dicho factor lo denominamos "Participación de los jóvenes en educación" y explica un $7 \%$ de la variabilidad total. Por último, el factor cinco (F5) al que denominamos "Formación permanente y formación superior de las empleadas", presenta correlaciones elevadas sólo con estas variables, siendo negativa la correspondiente al porcentaje de empleadas con educación superior (PESM). Este factor explica un 6\% de la variabilidad total.

Para completar este análisis representamos a los países que son objeto de 
estudio en los planos factoriales conjuntos F1-F2 y F1-F3 (Figuras 4 y 5$)^{11}$, que en conjunto explican casi un $80 \%$ de la variabilidad total. En dichas figuras observamos que, en general, la posición relativa de los países analizados en cuanto a educación y formación no es la misma si nos centramos en las mujeres que si lo hacemos en los hombres. Aunque, también es cierto, que en la mayor parte de estos países las diferencias entre estos grupos no es muy acentuada.

Figura 4

Países en el plano factorial 1-2

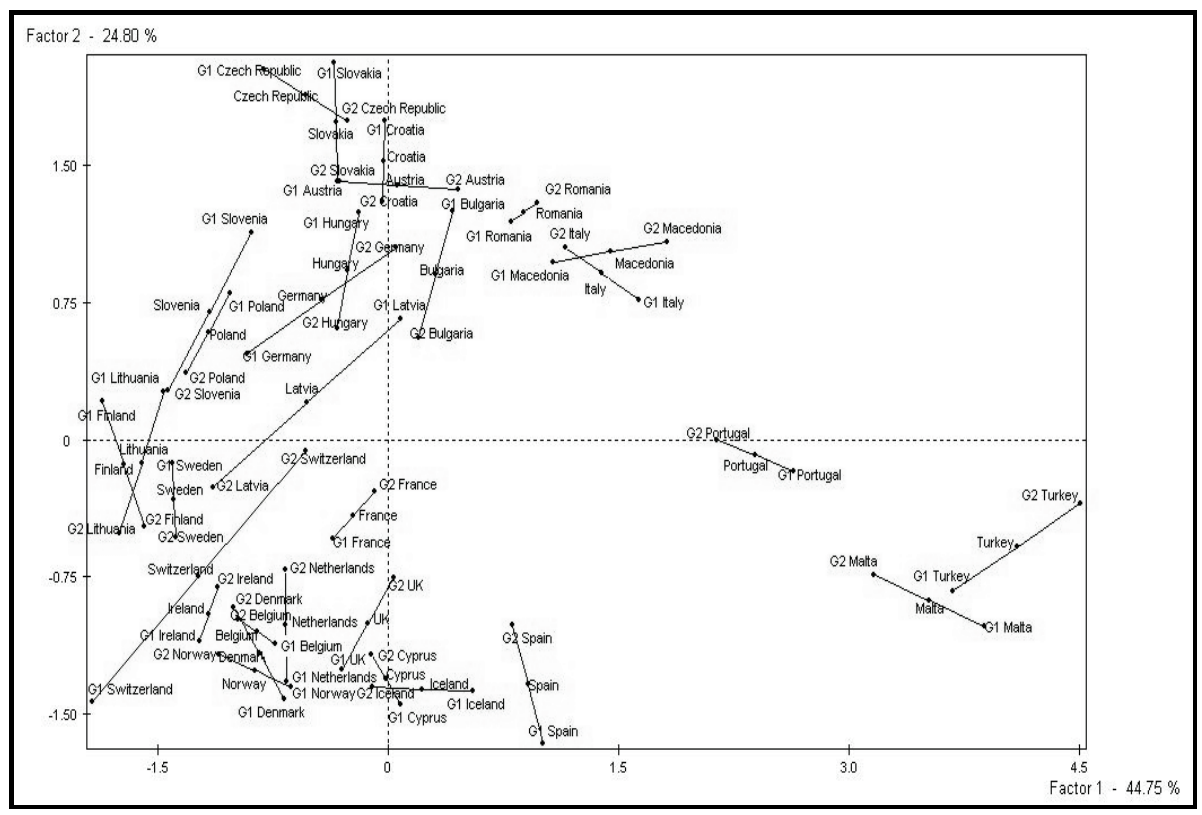

Fuente: Elaboración propia. G1=Hombres; G2=mujeres.

La representación en el plano factorial F1-F2 (Figura 4) destaca a Turquía, Malta, Portugal, Macedonia e Italia como los países con peor "Situación general en educación y formación" (F1), pero también presentan una situación peor que la media Rumanía, España, Bulgaria e Islandia. Asimismo, aunque los países mejor posicionados en este aspecto son Finlandia, Lituania y Suecia, destacamos a Suiza, Irlanda, Polonia y Eslovenia como países con situación bastante mejor que la media en lo relativo a este factor. En la figura anterior y en la

${ }^{11}$ La representación corresponde a la proyección en los planos factoriales de los países analizados desde un punto de vista medio (resumen de la información relativa a los dos grupos considerados) y desde un punto de vista parcial (posición relativa de cada país atendiendo a la educación $\mathrm{y}$ formación de hombres y mujeres por separado). 
Tabla 9 se puede observar, también, que los países que presentan las diferencias más acentuadas entre hombres y mujeres, en cuanto a este factor son Suiza, Letonia, Alemania, Turquía, Austria, Macedonia, Malta, Islandia, Eslovenia y República Checa.

En cuanto al factor que cuantifica la "Educación Superior" (F2), en la Figura 4 observamos que los países más deficitarios en este aspecto son Croacia, Eslovaquia y República Checa, seguidos muy de cerca por Austria, Rumanía, Macedonia, Italia, Hungría, Bulgaria y Alemania. En el polo opuesto, es decir, países que presentan niveles bastante superiores al medio en educación superior encontramos a España, Islandia, Noruega, Dinamarca, Bélgica, Chipre, Holanda, Reino Unido, Irlanda, Suiza y Malta. Centrándonos en las diferencias por razón de género, destacamos las que presentan Suiza, Letonia, Eslovenia, Lituania, Bulgaria, Finlandia, Eslovaquia, España, Hungría y Holanda (véanse la Tabla 9 y la Figura 4).

\section{Tabla 9}

Países con las mayores inercias intra

\begin{tabular}{|l|c|l|r|l|r|}
\hline \multicolumn{1}{|c|}{ Factor 1 } & Inercia & Factor 2 & Inercia & Factor 3 & Inercia \\
\hline Suiza & 20,98 & Suiza & 21,40 & Macedonia & 16,34 \\
Letonia & 16,07 & Letonia & 9,59 & Finlandia & 13,65 \\
Alemania & 10,23 & Eslovenia & 8,37 & Lituania & 10,95 \\
Turquía & 7,55 & Lituania & 6,69 & Chipre & 9,02 \\
Austria & 6,66 & Bulgaria & 5,47 & Turquía & 6,83 \\
Macedonia & 5,89 & Finlandia & 5,36 & Letonia & 6,50 \\
Malta & 5,58 & Eslovaquia & 4,82 & Bulgaria & 5,63 \\
Islandia & 4,68 & España & 4,77 & Dinamarca & 5,06 \\
Eslovenia & 3,22 & Hungría & 4,49 & Italia & 4,70 \\
República Checa & 3,14 & Holanda & 4,24 & Rumanía & 3,84 \\
\hline
\end{tabular}

Fuente: Elaboración propia.

Del análisis de la Figura 5, países en el plano factorial F1-F3, destacamos a Finlandia como el país que presenta la mejor posición en cuanto a "Participación y graduación en MCT". También en niveles bastante superiores a la media se encuentran Portugal, España y Suecia. Por el contrario, países como Holanda, Chipre, Letonia o Hungría se encuentran entre los más deficitarios en este aspecto. En lo que respecta a las diferencias más acentuadas entre hombres y mujeres, éstas son las que presentan Macedonia, Finlandia, Lituania, Chipre, Turquía, Letonia, Bulgaria, Dinamarca, Italia y Rumanía. 
Figura 5

Países en el plano factorial F1-F3

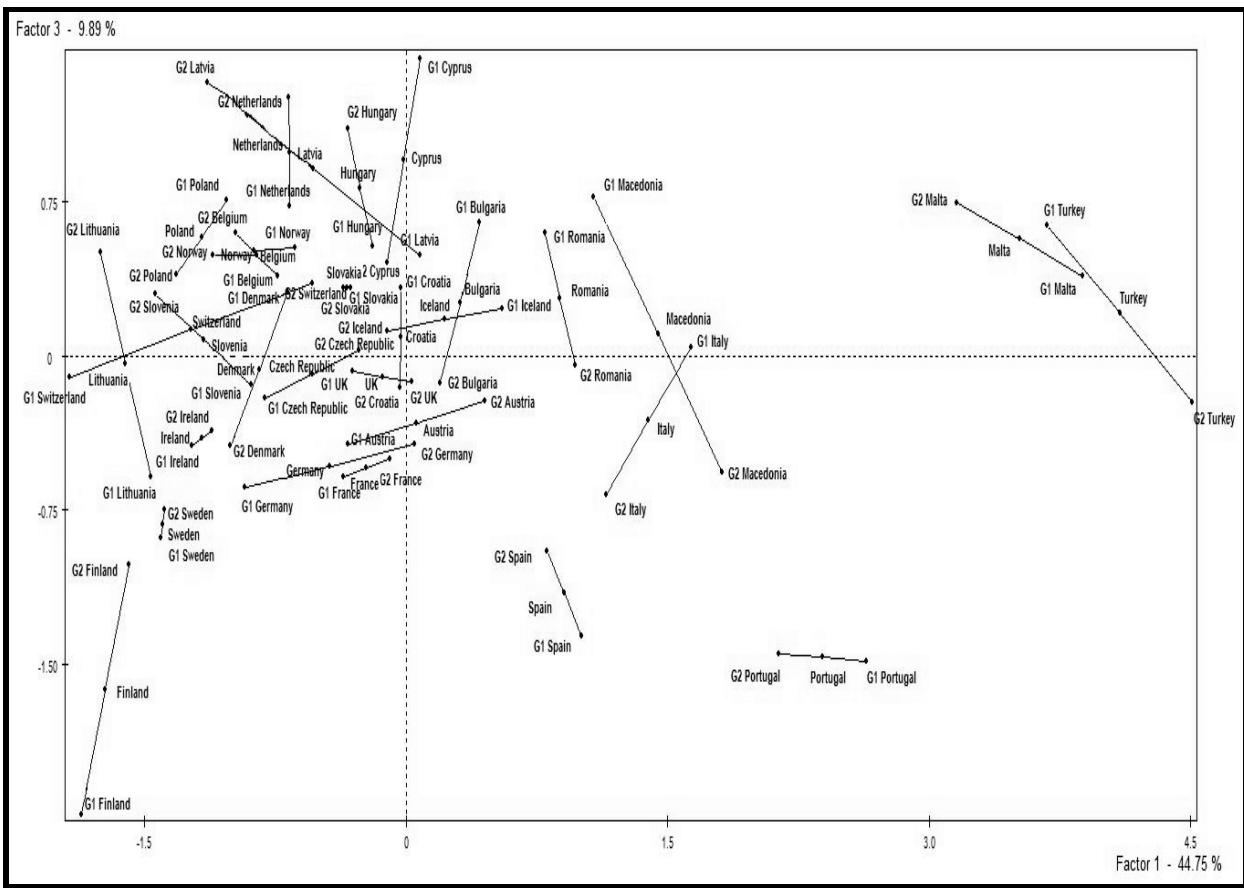

Fuente: Elaboración propia.

\section{LA SITUACIÓN EDUCATIVA Y EL CONTEXTO SOCIOECONÓMICO}

En esta sección complementamos el análisis previo sobre el estado de la educación en Europa con la información que, para la mayor parte de los países considerados en las secciones previas ${ }^{12}$, nos proporcionan diversos indicadores socioeconómicos. En este sentido, nos planteamos un doble objetivo. Por una parte, tratamos de establecer si existe relación entre la situación de estos países con respecto a las características socioeconómicas consideradas y su situación educativa, resumida esta última mediante los tres primeros factores del análisis factorial global. Por otra parte, y centrándonos en las diferencias de género, analizamos cómo de acentuadas son las diferencias entre hombres y mujeres en relación a las otras características socioeconómicas en aquellos países que presentan las mayores diferencias en educación.

${ }^{12}$ La no disponibilidad de información sobre algunas de estas variables en la fuente utilizada nos ha obligado a suprimir de este análisis posterior a Macedonia, Suiza y Turquía. 
Para conseguir el primero de los objetivos planteados, hemos aplicado el método estadístico de Análisis de Conglomerados. Con la aplicación de esta metodología pretendemos obtener grupos de países homogéneos con respecto a las características educativas y socioeconómicas que resumen los indicadores utilizados, pero de modo que países en grupos distintos difieran tanto como sea posible en relación a las mismas.

Las técnicas empleadas en este tipo de análisis pueden ser jerárquicas o no jerárquicas. Las primeras parten de considerar inicialmente tantos grupos como objetos. En el primer paso se agrupan los dos casos con menor distancia ${ }^{13}$ (o mayor similitud). En cada una de las sucesivas etapas, siguiendo el criterio de mínima distancia, se forma un nuevo grupo por unión de dos individuos aislados, de dos grupos, o por la adhesión de un individuo a un grupo ya formado en una etapa anterior. El proceso culmina con la obtención de un único grupo, que engloba a todos los individuos u objetos, formado por las aglutinaciones sucesivas, con niveles de similitud o distancia distintos ${ }^{14}$. La representación gráfica de estas etapas de formación de grupos, a modo de árbol invertido, se denomina dendograma.

Los métodos no jerárquicos, también conocidos como partitivos o de optimización, tienen el objetivo de formar clases excluyentes, en número fijado de por el investigador, de modo que sean homogéneas en su interior y con máxima divergencia entre ellas. Las clases que se forman constituyen una única partición y no están organizadas jerárquicamente ni relacionadas entre sí. La asignación de individuos a los grupos se hace mediante algún proceso que optimice el criterio de selección. Fijado el número de conglomerados a obtener, el primer paso es seleccionar una semilla, como centro inicial del conglomerado, y todos los elementos dentro de una distancia umbral que ha sido previamente especificada se incluyen dentro del conglomerado resultante. Entonces se selecciona otra semilla de conglomerado y la asignación continúa hasta que todos los elementos están distribuidos (Hair y otros; 2000). Los procedimientos de aglomeración no jerárquicos se denominan frecuentemente como aglomeración de Kmedias y difieren, esencialmente, en los criterios empleados para la selección de la semilla y la asignación de los elementos.

${ }^{13}$ La creación de grupos basados en la similitud de los elementos en ellos englobados exige una definición de este concepto, o de su complementario de "distancia" entre individuos. Existen diferentes formas de medir diferencias multidimensionales que, a su vez, proporcionan diversas posibilidades de análisis. La utilizada en este trabajo es la denominada distancia Euclídea, que mide la distancia entre dos elementos mediante la raíz cuadrada de la suma de los cuadrados de las diferencias entre los valores que cada variable toma en dichos elementos. Hair y otros (2000) indican que la elección de una u otra medida no influye significativamente en el resultado final del análisis.

${ }^{14}$ Este procedimiento jerárquico de formación de grupos es de tipo aglomerativo o ascendente. También existe el disociativo o descendente (véase González; 1991). 
En este trabajo hemos utilizado el método de aglomeración no jerárquico de K-medias. La aplicación de este método requiere decidir previamente el número de conglomerados a obtener y cuáles van a ser los centros o medias iniciales de los mismos (semillas). Esa decisión la hemos basado en los resultados obtenidos al aplicar el método jerárquico de aglomeración que resulta de combinar el algoritmo de Ward con la distancia euclídea (véase Uriel y Aldás, 2005). Las variables utilizadas para la clasificación han sido, además de las puntuaciones de estos países en los tres primeros factores del análisis global, que resumen la situación educativa de los mismos, las siguientes: Producto interior bruto per cápita a precios de mercado (PIBPC) ${ }^{15}$; Gasto público en educación como porcentaje del Producto interior bruto (GEPPIB) ${ }^{16}$; Tasa de paro en la población con edad inferior a veinticinco años (PJ); Tasa de paro global (PT); Porcentaje de personas en riesgo de pobreza o exclusión social entre las de dieciocho o más años $(\mathrm{PE})^{17}$ y Porcentaje del total de la población que se encuentra en riesgo de pobreza $(\mathrm{PM})^{18}$. La información utilizada, que procede también de Eurostat, ha sido la correspondiente al año $2010^{19}$.

El dendograma que resulta tras aplicar el método jerárquico a las variables tipificadas, que mostramos en la Figura 6, sugiere la existencia de entre seis y ocho grupos.

La implementación del método de aglomeración no jerárquico de K-medias con estas mismas variables y seis, siete y ocho grupos, utilizando como semillas iniciales las medias de los grupos obtenidos del método jerárquico, ha puesto de manifiesto la estabilidad de los resultados obtenidos en el análisis jerárquico (agrupaciones casi idénticas con uno y otro método, así como centros iniciales y finales muy próximos). Asimismo, el análisis de las variaciones experimentadas por la varianza residual al ir aumentando el número de grupos sugiere la idoneidad de la segmentación en ocho grupos.

\footnotetext{
${ }^{15}$ Gross domestic product (GDP) at market prices (euro per inhabitant).

${ }^{16}$ Total public expenditure on education as \% of GDP, for all levels of education combined.

${ }^{17}$ Son personas de dieciocho o más años que viven en hogares que cumplen, al menos, una de las siguientes condiciones: la renta equivalente disponible es inferior al $60 \%$ de la renta mediana nacional equivalente disponible; están severamente privados materialmente o con una intensidad laboral muy baja. (http://europa.eu/rapid/pressReleasesAction.do?reference=STAT/12/ 21\&type $=$ HTML).

${ }^{18}$ Porcentaje del total de la población con renta equivalente disponible inferior al $40 \%$ de la renta mediana nacional equivalente. http://epp.eurostat.ec.europa.eu/statistics_explained/index.php/ Glossary:At-risk-of-poverty_rate.

${ }^{19}$ Los datos relativos al PIBPC son del año 2009, que es el último disponible.
} 
Figura 6

Dendograma con el método de Ward y la distancia euclídea

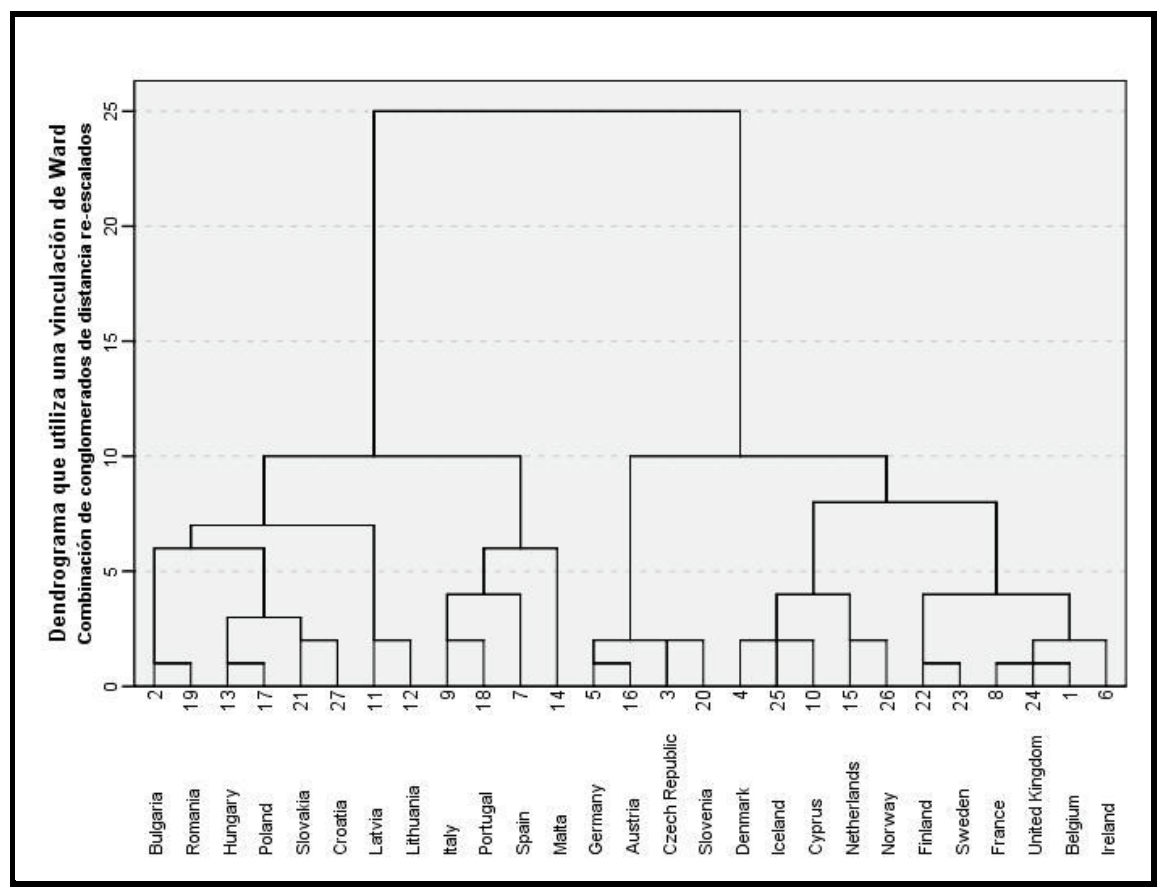

Fuente: Elaboración propia.

En la Tabla 10 presentamos la clasificación de los países atendiendo a esta agrupación y en la Figura 7 mostramos los valores medios de cada variable en los diferentes grupos, que nos permitirán analizar las principales características de los mismos. El análisis de los valores medios representados en la Figura 7 pone de manifiesto que los países clasificados en los grupos uno y dos (noroeste de Europa con excepción de Chipre) son los que presentan mejor situación en cuanto a educación y a los indicadores socioeconómicos analizados se refiere. Si bien los países del grupo dos son deficitarios en cuanto a "Participación y graduación en MCT" (F3), en lo relativo a las restantes características destacamos que ambos presentan buena "Situación general en educación y formación" (F1) y buen nivel de "Formación en educación superior" (F2). Tanto el nivel medio del Producto interior bruto per cápita (PIBPC) como el correspondiente al porcentaje del Producto interior bruto que representa el gasto en educación (GEPPIB) son muy superiores a la media global, destacando el grupo dos como el que destina un mayor porcentaje del Producto interior bruto a educación. En cuanto a paro (PJ y PT), los valores medios del grupo uno son muy similares a la media global, mientras que el grupo dos es el que presenta los niveles medios 
más bajos en estas variables. Así mismo, en lo relativo a pobreza y exclusión social (PE y PM) en ambos grupos se alcanzan niveles inferiores a la media.

Tabla 10

Composición de los diferentes grupos

\begin{tabular}{|c|l|}
\hline Grupos & \multicolumn{1}{|c|}{ Países } \\
\hline 1 & Bélgica, Francia, Reino Unido, Irlanda, Finlandia y Suecia \\
\hline 2 & Dinamarca, Islandia, Chipre, Holanda y Noruega \\
\hline 3 & República Checa, Eslovenia, Alemania y Austria \\
\hline 4 & Bulgaria y Rumanía \\
\hline 5 & Hungría, Polonia, Eslovaquia y Croacia \\
\hline 6 & Letonia y Lituania \\
\hline 7 & España, Italia y Portugal \\
\hline 8 & Malta \\
\hline
\end{tabular}

Fuente: Elaboración propia.

Por el contrario, los grupos peor posicionados son el cuatro y el seis. Ambos comparten las puntuaciones medias más elevadas en pobreza monetaria (PM), siendo en el conglomerado cuatro (Bulgaria y Rumanía) donde se alcanza la mayor puntuación media en pobreza y exclusión (PE) de todos los grupos analizados, seguido a corta distancia por el grupo seis. Este último grupo (Letonia y Lituania) también presenta las mayores tasas de paro, tanto juveniles como globales (PJ y PT); sin embargo, se da la paradoja de que su nivel medio en déficit formativo (F1) es el menor de los analizados, el nivel de formación en educación superior (F2) es similar al medio global y la partida que el estado dedica a educación, medida en porcentaje del PIB, es también similar a la media global. Esto es, a pesar de tener un nivel medio muy bajo en el Producto interior bruto per cápita, se realiza un gran esfuerzo en educación y se consiguen niveles aceptables de formación que coexisten con altas tasas de paro y pobreza. En Bulgaria y Rumanía (grupo cuatro) con un nivel medio de Producto interior bruto per cápita similar al del grupo seis, pero poco esfuerzo en educación en proporción a éste, se observa un gran déficit formativo en los distintos niveles considerados que va acompañado, sin embargo, de tasas de paro similares a la media global.

En general, podemos afirmar que los países con los niveles más altos de producto interior bruto per cápita (PIBPC) son los que destinan una mayor parte de su riqueza a educación y ello se corresponde con una buena situación educativa en los distintos niveles considerados que va acompañada de bajos niveles de paro y pobreza. Sin embargo, entre los de menor PIBPC, el esfuerzo que se realiza en educación no parece garantizar, en general, una buena situación en educación. Por otra parte, tampoco se puede afirmar que, transversalmente, el 
déficit formativo condicione los niveles de paro o pobreza del país (véanse las características del grupo unitario formado por Malta, las del grupo cuatro o las del grupo siete).

Figura 7

Valores medios de los indicadores en los diferentes grupos

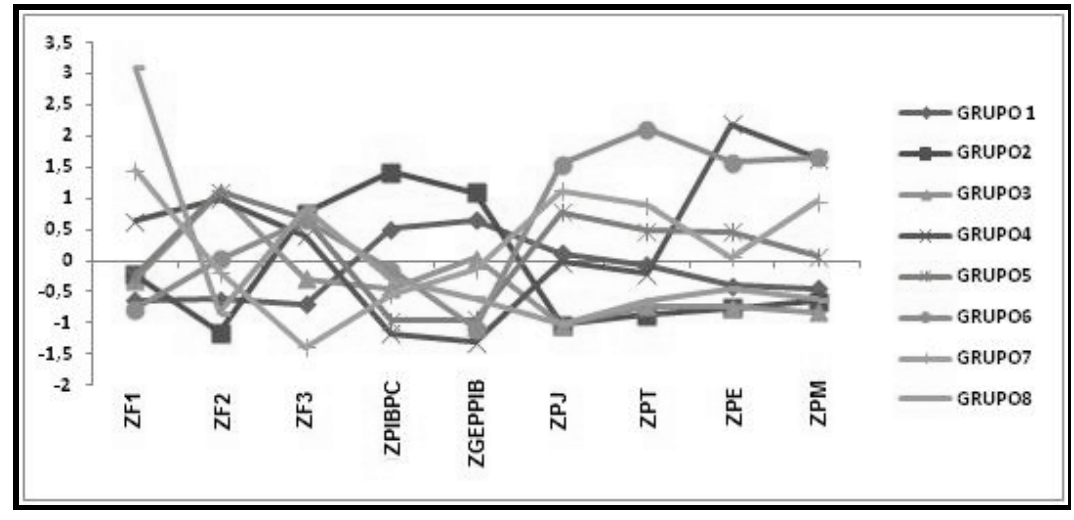

Fuente: Elaboración propia.

Por último, en relación al grupo en el que queda clasificada España (grupo siete), constituido por Italia, España y Portugal (parte del sur de Europa), se observan altas tasas de paro, sólo superadas por Letonia y Lituania, y niveles de pobreza monetaria bastante elevados, que coexisten con un nivel medio de Producto interior bruto per cápita (PIBPC) y un esfuerzo público en educación (GEPPIB) inferior al medio. Estos países presentan un nivel medio muy elevado de déficit formativo general (F1), que sólo es superado por Malta y Turquía. En contrapartida, el nivel de formación en educación superior (F2) en España y Portugal es superior a la media del grupo de países analizados y en participación y graduación en Matemáticas, Ciencias y Tecnología (F3) los tres alcanzan niveles superiores al medio ${ }^{20}$.

Concluimos esta sección con el análisis relativo a las diferencias socioeconómicas entre hombres y mujeres en los países que presentan las mayores diferencias de género en lo que a educación se refiere, segundo de los objetivos planteados en este apartado. Para esta finalidad, además de aquellas características incluidas en el análisis previo para las que tiene sentido la desagregación

${ }^{20}$ Como se indicaba en Marcenaro y Navarro (2001), la gran expansión de la educación superior en España, comparada con los países de su entorno, puede ser debida al intento por parte de los jóvenes de superar las elevadas tasas de paro, así como al aumento de la oferta educativa y de las facilidades para acceder al sistema público de becas. 
por género (PJ, PT, PE y PM), hemos considerado la brecha salarial ${ }^{21}$ (BS). En este sentido, destacamos que, entre los países que presentan las diferencias ${ }^{22}$ más acentuadas en cuanto a su situación general en educación y formación (F1) (véase la Tabla 9), Alemania, Austria y República Checa se encuentran a la cabeza de los países europeos con más brecha de género positiva en los salarios en 2010 y Suiza y Letonia están por encima de la media europea en dicho aspecto. Al mismo tiempo, en Austria y República Checa observamos también las mayores diferencias positivas en relación a pobreza y exclusión social (PE), siendo también de las más altas en lo relativo a pobreza monetaria (PM). En el resto de los países mencionados observamos diferencias bastante por encima de la media (UE-27) en cuanto a pobreza y exclusión pero, de ellos, sólo en Suiza observamos una diferencia superior a la media en pobreza monetaria. Con respecto a las diferencias de género en las tasas de paro (PJ y PT) en este grupo de países, sólo observamos más paro en mujeres que en hombres en República Checa.

Por último, entre los países con mayores diferencias de género en los factores relativos a educación superior (F2 y F3), Bulgaria, Finlandia, Letonia y Lituania, destacamos que en todos ellos las tasas de paro son superiores entre los hombres que entre las mujeres, tanto si nos restringimos al paro en los menores de veinticinco años como si consideramos el paro total. En relación a las diferencias en pobreza monetaria (PM) sólo observamos un valor por encima del medio de la Unión Europea en Bulgaria. Sin embargo, en todos ellos las diferencias en pobreza y exclusión se sitúan por encima de la media de la Unión Europea y la brecha salarial (BS) sólo es superior a la media europea en Finlandia y Letonia.

\section{CONCLUSIONES}

De la descripción unidimensional concluimos que, para el conjunto de países analizados, el nivel de formación de la mujer joven es ligeramente superior al del hombre y también es mayor su participación en educación. Entre la población adulta (de 25 a 64 años), aunque el porcentaje de mujeres con nivel de estudios inferior a segunda etapa es ligeramente superior al existente entre los hombres, cuando nos centramos en la formación superior, en la participación en actividades de educación y/o formación o en el nivel de formación de las personas empleadas, las diferencias vuelven a ser favorables a las mujeres. Sin em-

${ }^{21}$ Gender pay gap in unadjusted form in \% (NACE Rev. 2: Industry, construction and services, except public administration, defense, compulsory social security).

${ }^{22}$ En lo que sigue, salvo que indiquemos lo contrario, cuando hablamos de diferencias estamos comparando, en términos relativos, los niveles asociados a las mujeres con los correspondientes a los hombres. Ello significa que las diferencias positivas representan que, para el correspondiente indicador, el valor observado para el hombre es superior al de la mujer. 
bargo, en el campo de Matemáticas, Ciencias y Tecnología las tasas de participación y graduación de las mujeres son muy inferiores a las de los hombres.

De la aplicación del AFM a las variables educativas desglosadas por género concluimos que existe una relación bastante acentuada entre el grupo de variables que describe la situación en educación y formación de los hombres y el correspondiente a las mujeres, así como que los aspectos comunes a ambos grupos quedan bien representados por los tres primeros factores del análisis global que, en conjunto, explican casi un $80 \%$ de la variabilidad global. De acuerdo con las variables que con ellos presentan las correlaciones más altas, a estos factores los hemos denominado "Situación general en educación y formación" (F1), "Formación en educación superior" (F2) y "Participación y graduación en MCT" (F3), respectivamente. Del análisis de las puntuaciones de los países en estos factores destacamos que los únicos países deficitarios en todos los aspectos de la educación considerados en este estudio son Bulgaria, Macedonia y Rumanía y, en cuanto a las diferencias de género que en ellos observamos, Macedonia presenta diferencias acentuadas en los factores uno y tres; Bulgaria en los factores dos y tres y Rumanía sólo en el factor tres. En el lado opuesto, es decir, países bien situados en cuanto a todos los factores, encontramos a Dinamarca, Finlandia, Francia, Irlanda, Lituania, Reino Unido y Suecia. De ellos, en Irlanda, Reino Unido y Suecia observamos poca diferencia entre hombres y mujeres.

En relación al grupo en el que queda clasificada España (grupo siete), constituido por Italia, España y Portugal (parte del sur de Europa), se observan altas tasas de paro, que sólo superan Letonia y Lituania, y niveles de pobreza monetaria bastante elevados, que coexisten con un nivel medio de Producto interior bruto per cápita (PIBPC) y un esfuerzo público en educación (GEPPIB) inferior al medio. Estos países presentan una situación general en educación muy inferior a la media (F1), que sólo es superada por Malta y Turquía. En contrapartida, el nivel de formación en educación superior (F2) en España y Portugal es superior a la media del grupo de países analizados y en cuanto a participación y graduación en Matemáticas, Ciencias y Tecnología (F3) los tres alcanzan niveles superiores al medio.

Por último, podemos afirmar que, en el contexto analizado, los países con los niveles más altos de producto interior bruto per cápita (PIBPC) son los que destinan una mayor parte de su riqueza a educación, lo que se corresponde con una buena situación educativa en los distintos niveles considerados que va acompañada de bajos niveles de paro y pobreza. Sin embargo, entre los de menor PIBPC, el esfuerzo que se realiza en educación parece no garantizar, en general, una buena situación educativa y tampoco podemos afirmar que, transversalmente, el déficit formativo esté asociado a altos niveles de paro o pobreza 
(véanse las características del grupo unitario formado por Malta, las del grupo cuatro o las del grupo siete).

\section{REFERENCIAS BIBLIOGRÁFICAS}

ABASCAL, E., FERNÁNDEZ, K., MODROÑO, J.I. Y LANDALUCE, M.I. (2001): "Técnicas factoriales de análisis de tablas múltiples: Nuevos desarrollos empíricos", Documentos de Trabajo BILTOKI, ISSN 1134-8984, № 6.

ABASCAL, E., GARCÍA, I. Y LANDALUCE, M.I. (2006): "Trayectorias de las Comunidades Autónomas según sus tasas de paro por categorías de edad. Un análisis de tablas tridimensionales", Estadística Española, 48 (163), pp. 525-550.

ABASCAL, E. Y LANDALUCE, M. I. (2002): “Análisis Factorial Múltiple como técnica de estudio de la estabilidad de los resultados un análisis de Componentes Principales", Quëstiió, 26 (1-2), pp.109-122.

COMISIÓN EUROPEA (CE) (1999): Informe anual de la Comisión: Igualdad de oportunidades entre mujeres y hombres en la Unión Europea - 1998, COM 99-0106 final. http://eurlex.europa.eu/LexUriServ/LexUriServ.do?uri=CELEX: 51999DC0106:ES:HTML.

COMISIÓN EUROPEA (CE) (2002): Comunicación de la Comisión al Consejo, al Parlamento Europeo, al Comité Económico y Social y al Comité de las Regiones - Integración de la igualdad entre hombres y mujeres en los documentos de programación de los Fondos Estructurales para 2000-2006, COM/2002/0748 final. http://eurlex.europa.eu/Notice.do?val=267156:cs\& lang=es\&list=267156:cs,\&pos=1\&page=1\&nbl=1\&pgs=10\&hwords.

COMISIÓN EUROPEA (CE) (2005): Comunicación al Consejo Europeo de Primavera-Trabajando juntos por el crecimiento y el empleo-Relanzamiento de la Estrategia de Lisboa, COM/2005/24final. http://europa.eu/legislation summaries/employment_and_social_policy/eu2020/growth_and_jobs/c1132 5_es.htm

COMISIÓN EUROPEA (CE) (2007): Comunicación de la Comisión Un marco coherente de indicadores y puntos de referencia para el seguimiento de los avances hacia los objetivos de Lisboa en el ámbito de la educación y la formación, COM/2007/0061 final. http://eurlex.europa.eu/Notice.do?val= 443773:cs\&lang=es\&list=446461:cs,443773:cs, \&pos=2\&page=1\&nbl=2\&pgs $=10 \&$ hwords.

COMISIÓN EUROPEA (CE) (2010): Europa 2020 Una estrategia para un crecimiento inteligente, sostenible e integrador, COM/2010/2020 final. http://eurlex.europa.eu/LexUriServ/LexUriServ.do?uri=CELEX:52010DC2020: ES:NOT. 
CONSEJO EUROPEO (C) (2009): Conclusiones del Consejo, de 12 de mayo de 2009, sobre un marco estratégico para la cooperación europea en el ámbito de la educación y la formación (ET 2020), 2009/C 119/02. http://eurlex.europa.eu/LexUriServ/LexUriServ.do?uri=OJ:C:2009:119:0002: 0010:ES:PDF.

EUROPEAN COMMUNITY TREATY (ECT) (1957): Treaty Establishing the European Community, Rome, 25 March 1957. http://www.hri.org/docs/ Rome57/.

ESCOFIER, B. Y PAGES, J. (1990): Analyses factorielles simples et multiples. Paris: Dunod.

ESCOFIER, B. Y PAGÈS, J. (1992): Análisis Factoriales simples y múltiples: Objetivos, Métodos e interpretación. Bilbao: Servicio Editorial de la Universidad del País Vasco.

ESCOFIER, B. Y PAGĖS, J. (1994): "Multiple factor analysis (AFMULT package)", Computational Statistics and Data Analysis, 18, pp.121-140.

ESCOUFIER, Y. (1973): "Le traitement des variables vectorielles". Biometrics, 29, pp.751-760.

GARCÍA LAUTRE, I. Y ABASCAL FERNÁNDEZ, E. (2003): "Una metodología para el estudio de la evolución de variables latentes. Análisis de las infraestructuras de carreteras de las comunidades autónomas (1975-2000)", Estadística Española, 45 (153), pp.193-210.

GONZÁLEZ LÓPEZ-VALCÁRCEL, B. (1991): Análisis multivariante. Aplicación al ámbito sanitario, Barcelona, SG editores, S.A.

JOSSE, J., PAGĖS, J. AND HUSSON, F. (2008): "Testing the significance of the RV coefficient", Computational Statistics and Data Analysis, 53, pp. 8291.

HAIR, J.F.; ANDERSON, R.E.; TATHAM, R.L. Y BLACK, W.C. (2000): Análisis Multivariante, Madrid, Prentice Hall Iberia.

KAISER, H.F. (1960): "The Application of Electronic Computers to Factor Analysis", Educational and Psychological Measurement, 10, pp. 141-151.

LEBART, L., MORINEAU, A. Y PIRON M. (1995): Statistique Exploratoire Multidimensionnelle. París: Dunod.

LYNCH, K. AND FEELEY, M. (2009): Gender and education (and employment): gendered imperatives and their implications for women and men: lessons from research for policy makers, Technical Report, NESSE Network of Experts, ISBN 978-92-79-12964-3.http://hdl.handle.net/10197/2467.

MARCENARO GUTIÉRREZ, O.D. Y NAVARRO GÓMEZ, M.L. (2001): "Un análisis microeconómico de la demanda de educación superior en España", Estudios de Economía Aplicada, 19, pp. 69-86.

ORGANIZACIÓN DE LAS NACIONES UNIDAS PARA LA EDUCACIÓN, LA CIENCIA Y LA CULTURA (UNESCO) (2000): Igualdad y Equidad de género: Revisión de los objetivos alcanzados por la UNESCO desde la Cuarta 
Conferencia Mundial sobre las Mujeres (Pekín, 1995). Unidad para la Mejora del Estatus de las Mujeres y la Igualdad de Género, UNESCO.

PAGES, J. (1998): "Multiple factor analysis". En A. Morineau, K. Fernández Aguirre (Eds.): Analyses Multidimensionnelles des données, ANGUS 97" (pp. 38-48). CISIA-CERESTA.

PAGĖS, J. (2004): "Multiple Factor Analysis: Main Features and Application to Sensory Data", Revista Colombiana de Estadística, 27 (1), pp. 1-26.

RED EURYDICE (2010): Diferencias de género en los resultados educativos: medidas adoptadas y situación actual en Europa, ISBN 978-92-9201-131-4. http://www.educacion.es/cide/eurydice.

UNIÓN EUROPEA (UE) (2010): Versión consolidada del Tratado de funcionamiento de la Unión Europea, Diario Oficial de la Unión Europea, C 83/47. http://eur-lex.europa.eu/JOHtml.do?uri=OJ:C:2010:083:SOM:ES:HTML.

URIEL, E. Y ALDÁS, J. (2005): Análisis Multivariante Aplicado, Madrid, Eds. Thomson, Paraninfo S.A. 
